\title{
Design of a Cross-Slot Flow Channel for Extensional Viscosity Measurements
}

\author{
Manuel A. Alves \\ Faculdade de Engenharia da Universidade do Porto, Departamento de Engenharia Química, CEFT, \\ Rua Dr. Roberto Frias s/n, 4200-465 Porto, Portugal
}

\begin{abstract}
In this work we present an algorithm for optimal shape design of viscoelastic fluid flow. A finite-volume viscoelastic code is coupled with the CONDOR optimizer [1] and an automated mesh generation and adaptation procedure, in order to find the shape of a given flow geometry, capable of achieving optimal performance. As an example of application we design an optimized cross-slot microfluidic chip appropriate for measuring the extensional viscosity of dilute polymeric solutions. The microfluidic cross-slot flow geometry is able to generate homogeneous elongational flow with regions of constant strain-rate, a requirement to produce meaningful rheological measurements.
\end{abstract}

Keywords: Optimal Shape Design; Cross-Slot; Extensional Viscosity; Microfluidics; Viscoelastic Fluid Flow. PACS: 02.60.Pn, 47.50.-d, 47.11.Df, 83.85.Cg, 83.85.Rx

\section{INTRODUCTION}

Optimal shape design (OSD) tools are useful for a wide variety of applications, ranging from fluid mechanics to electromagnetism or structure mechanics, or even to a combination of the three [2]. The development of optimization methods for CFD studies requires three major components [3]: (i) a solver of the nonlinear optimization problem, defined by an objective function and the functional constraints; (ii) a method for modeling the flow geometry based on the design variables, and automatic generation of the mesh; (iii) a CFD flow solver that evaluates the objective function and the functional constraints. CFD based optimization tools are valuable for various engineering applications, although are also very demanding in terms of computational resources because each evaluation of the objective function requires a full CFD simulation.

As a practical application of the OSD method, we present results for the optimal design of a microfluidic rheometer-on-a-chip, appropriate for extensional viscosity measurements of dilute polymeric solutions. The focus of this study will be on cross-slot flow devices, and the goal is to find the best design of the shape of the flow geometry in order to achieve optimal performance, which corresponds to an ideal planar extensional flow.

\section{NUMERICAL METHOD}

The optimal shape design methodology proposed includes a fully-automated mesh generator program, a viscoelastic fluid flow solver [4,5] and the CONDOR [1] optimizer. The selection of the derivative-free CONDOR optimizer was motivated by its easy implementation and for being freely available. Starting from an initial estimate of the design variables, $\mathbf{X}^{\mathbf{0}}$, that describe the shape of the flow geometry, the initial mesh is generated and the CFD simulation is undertaken. From the numerical solution the objective function is evaluated, and this information is sent to the CONDOR optimizer. This process is repeated for a number of design variable parameters, supplied by the optimizer, until the optimal shape is found. A diagram of the optimization procedure is presented in Figure 1(a). We use B-splines in order to describe the flow geometry using a total of 9 adjustable parameters (the knots that define the B-spline). Additional calculations using 19 adjustable parameters were also undertaken, but the results obtained were similar.

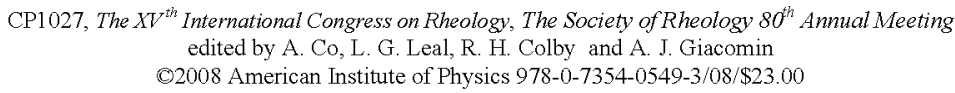


Due to the symmetry of the flow geometry, only one quarter (the first quadrant) of the cross-slot geometry is optimized, and the remaining walls are obtained assuming geometrical symmetry. The initial estimate corresponds to a rounded cross-slot geometry, which is illustrated in Figure 1(b).

The CFD flow solver employed is a finite-volume code developed for simulation of laminar flows of viscoelastic fluids described by differential-type constitutive equations [4,5]. The governing equations are those expressing conservation of mass, $\nabla \cdot \mathbf{u}=0$, and linear momentum,

$$
-\nabla p+\nabla \cdot \boldsymbol{\tau}=\mathbf{0}
$$

under creeping flow conditions. The constitutive equation selected was the upper-convected Maxwell (UCM) model,

$$
\boldsymbol{\tau}+\lambda\left(\frac{\partial \boldsymbol{\tau}}{\partial t}+\nabla \cdot \mathbf{u} \boldsymbol{\tau}\right)=\eta\left(\nabla \mathbf{u}+\nabla \mathbf{u}^{\mathrm{T}}\right)+\lambda\left(\boldsymbol{\tau} \cdot \nabla \mathbf{u}+\nabla \mathbf{u}^{\mathrm{T}} \cdot \boldsymbol{\tau}\right)
$$

where $\lambda$ and $\eta$ are the relaxation time and the shear viscosity of the fluid, respectively. The numerical technique is described in detail in $[4,5]$. The flow solver works as a black-box in the optimization cycle.
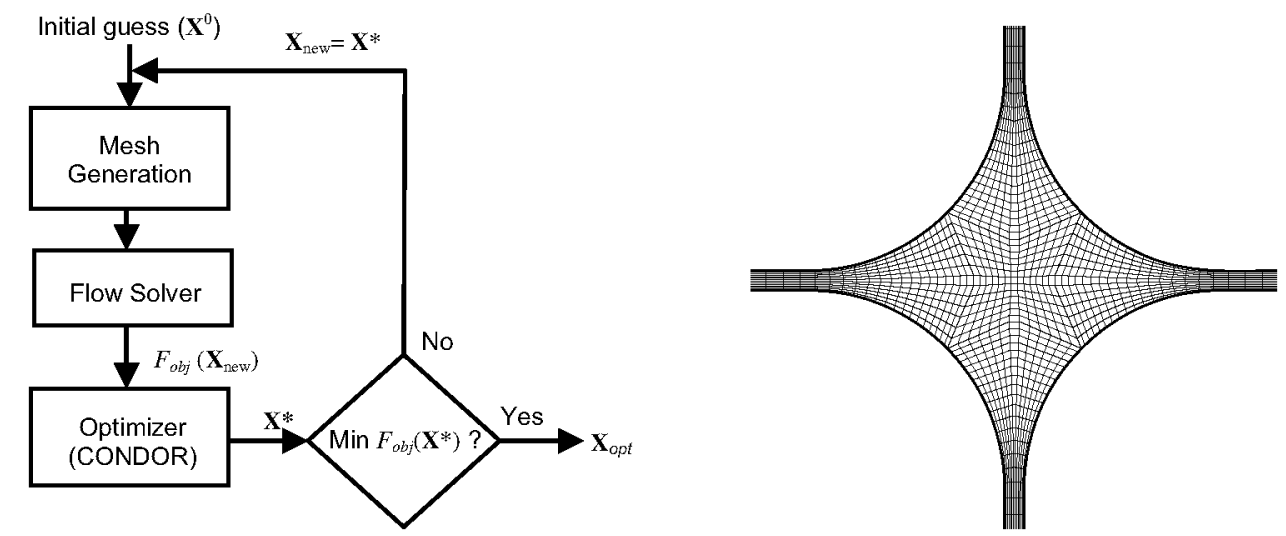

FIGURE 1. (a) Schematic illustration of the optimization cycle and (b) zoomed view of the coarse mesh used as initial estimate.

\section{RESULTS AND DISCUSSION}

We start by analyzing the effect of the Deborah number $(D e=\lambda U / H)$ upon the shape of the optimized cross-slot geometry. The characteristic velocity used in the definition of $D e$ is the average velocity in each channel, $U$, with minimum half-width $H$. In Figure 2 we compare the optimal shape meshes and the velocity profiles obtained for a Newtonian fluid and a UCM fluid at $D e=4(\lambda \dot{\varepsilon}=0.4)$. The similarity between both results is remarkable, thus allowing the design of a cross-slot geometry capable of achieving optimal performance for a wide range of flow conditions and fluids.

The predicted streamlines and normal stresses are illustrated in Figure 3 for the viscoelastic case. The typical birefringence strand, characteristic of viscoelastic fluid flows, is clearly visible in Figure 3 (b) along the vertical centerline. Most of the simulations were done in coarse meshes (M1), composed of 1521 cells, as illustrated in Figure 2 (a), (b). A limited number of simulations with a refined mesh (M2), with a total of 5825 cells, were also undertaken, but the results are similar to those obtained in the coarse mesh, as shown in Figure 2 (c) and (d), thus justifying the use of the coarse meshes in the remaining simulations.

Using the optimized geometry obtained for Newtonian fluid flow we found that the proposed design of the cross-slot geometry generates a velocity field that is practically insensitive to inertial and elastic effects. This feature was confirmed for a broad range of operating flow conditions and for the constitutive equations tested, namely the upper-convected Maxwell, Oldroyd-B and Phan-Thien-Tanner models. In particular, the optimized device was found to operate efficiently under creeping flow conditions, thus making the proposed design suitable for microfluidic applications, for which the elastic effects are enhanced due to the small dimensions and high strain-rates typically observed.

\section{ACKNOWLEDGMENTS}

The authors acknowledge the financial support from FEDER and FCT through projects POCI/EQU/56342/2004 and PTDC/EQU-FTT/71800/2006. 


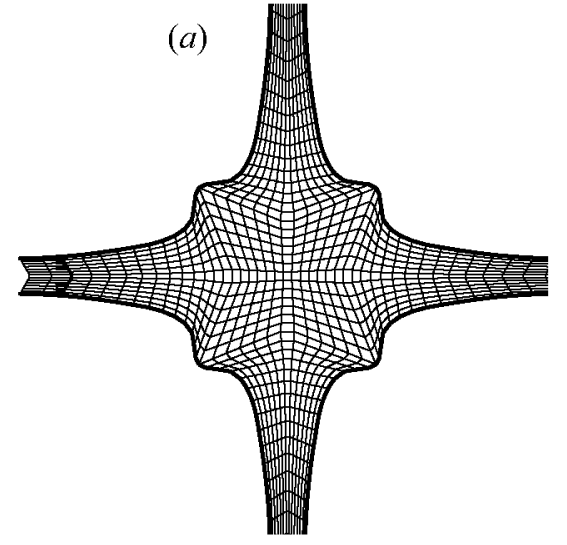

(c)

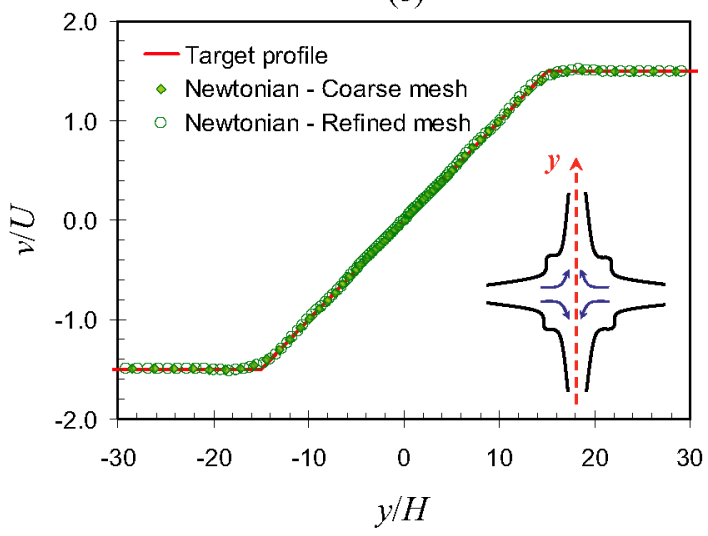

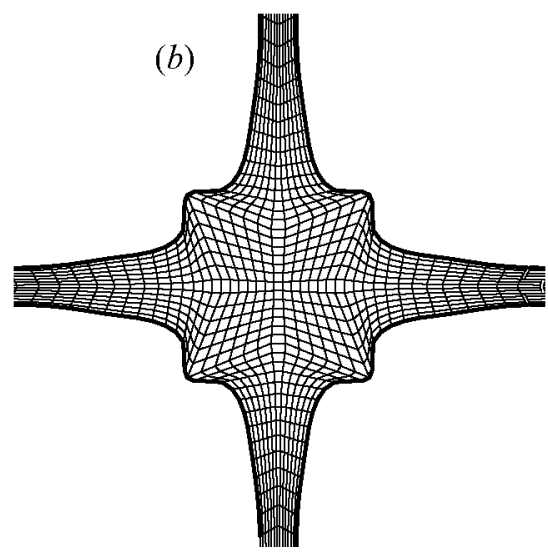

(d)

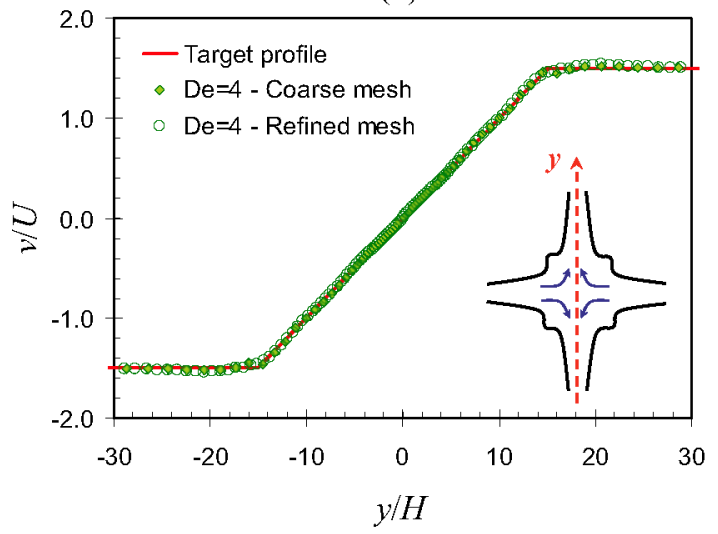

FIGURE 2. Optimized meshes calculated for (a) Newtonian fluid and (b) UCM fluid at $D e=4$. Velocity profiles along the vertical centerline $(x=0)$ for the optimized cross-slot flow geometries: (c) Newtonian fluid; (d) UCM fluid at $D e=4$.
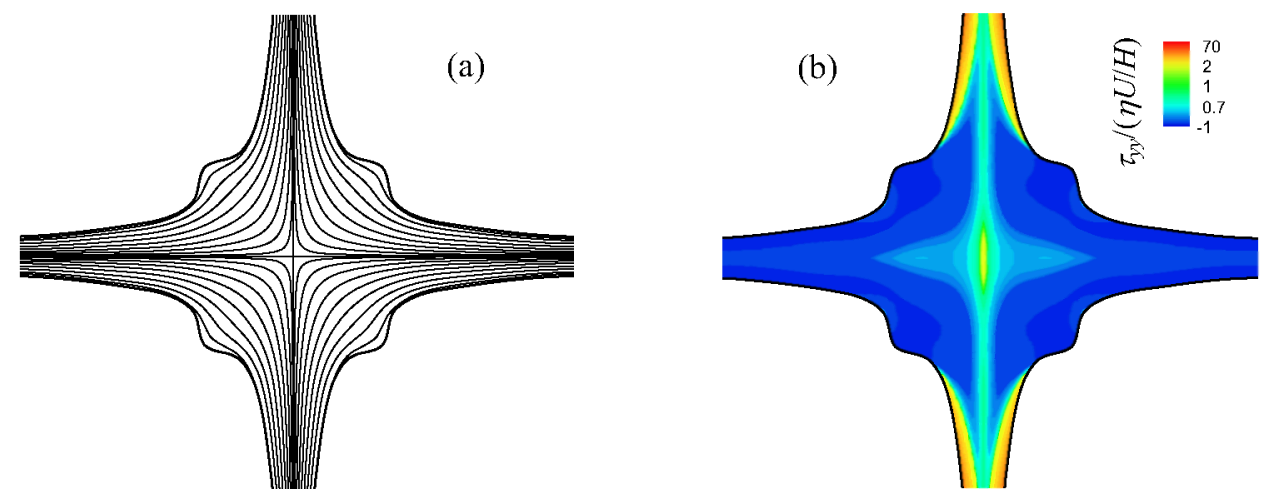

FIGURE 3. (a) Predicted streamlines and (b) contour plot of $\tau_{y y} /(\eta U / H)$ for a UCM fluid at $D e=4$.

\section{REFERENCES}

1. F.V. Berghen and H. Bersini, J. Comp. Appl. Math. 181, 157-175 (2005).

2. B. Mohammadi, and O. Pironneau, Ann. Review Fluid Mech. 36, 255-279 (2004).

3. Y. Tahara, S. Tohyama and T. Katsui, Int. J. Num. Meth. Fluids 52, 499-527 (2006).

4. P.J. Oliveira, F.T. Pinho, G.A. Pinto, J. Non-Newt. Fluid Mech., 79, 1 (1998).

5. M.A. Alves, P. J. Oliveira and F. T. Pinho, Int. J. Num. Meth. Fluids 41, 47-75 (2003). 